\title{
ON THE DETERMINACY OF COMPLEX JACOBI MATRICES
}

\author{
BERNHARD BECKERMANN and MIRTA CASTRO SMIRNOVA*
}

\begin{abstract}
The aim of this paper is to explore to which extend the theory of the Hamburger moment problem for real Jacobi matrices generalizes to the case of complex Jacobi matrices. In particular, we characterize the indeterminacy in terms of uniqueness of closed extensions of Jacobi matrices, and discuss the link to the growth of the smallest singular values of the underlying Hankel matrices. As a byproduct, we give a positive answer to the open question whether determinacy is preserved under bounded perturbations.
\end{abstract}

\section{Introduction}

Given $a_{n} \in \mathrm{C} \backslash\{0\}$ and $b_{n} \in \mathrm{C}$, we consider the following complex Jacobi matrix

$$
\mathscr{A}=\left(\begin{array}{cccc}
b_{0} & a_{0} & 0 & \ldots \\
a_{0} & b_{1} & a_{1} & \ddots \\
0 & a_{1} & b_{2} & \ddots \\
\vdots & \ddots & \ddots & \ddots
\end{array}\right)
$$

We denote by $\ell^{2}$ the Hilbert space of complex square-summable sequences, with the usual scalar product $(u, v)=\sum \overline{u_{j}} v_{j}$. Furthermore, for a linear operator $T$ in $\ell^{2}$, we denote by $\mathscr{D}(T), \mathscr{R}(T), \mathscr{N}(T)$, and $\sigma(T)$ its domain of definition, its range, its nullspace, and its spectrum, respectively. In what follows we identify $\mathscr{A}$ with the operator acting on the set $\mathscr{C}_{0}$ of finite linear combinations of the canonical vectors $e_{0}, e_{1}, \ldots \in \ell^{2}$.

Complex Jacobi matrices occur naturally in the study of formal orthogonal polynomials and Jacobi continued fractions: define the sequences of polynomials $p(z):=\left(p_{n}(z)\right)_{n \geq 0}$ and $q(z):=\left(q_{n}(z)\right)_{n \geq 0}$ as solutions of the recurrence

${ }^{*}$ B. B. is supported in part by INTAS project 2000-272, and in part by the Ministry of Science and Technology (MCYT) of Spain and the European Regional Development Fund (ERDF) through the grant BFM2001-3878-C02-02. M. C. S. is on leave from Havana University. Supported in part by INTAS project 2000-272, and by a grant from the French-Russian university network.

Received November 20, 2002; in revised form July 8, 2003. 
relation

$$
a_{n} y_{n+1}+b_{n} y_{n}+a_{n-1} y_{n-1}=z y_{n}, \quad n=0,1,2, \ldots,
$$

with initial conditions

$$
\left\{\begin{aligned}
q_{-1}(z) & =0, \quad q_{0}(z)=1, \\
p_{0}(z) & =0, \quad p_{1}(z)=1 / a_{0} .
\end{aligned}\right.
$$

Then $\left(p_{n}(z) / q_{n}(z)\right)_{n \geq 0}$ is a sequence of convergents of the Jacobi continued fraction associated with $\mathscr{A}$. Moreover, $\left(q_{n}(z)\right)_{n \geq 0}$ is a sequence of formal orthogonal polynomials with respect to the linear functional $c$ acting on the set of polynomials via

$$
c_{n}:=c\left(x^{n}\right)=\left(e_{0}, \mathscr{A}^{n} e_{0}\right), \quad n \geq 0 .
$$

It is known that the corresponding sequence of Hankel matrices

$$
H_{n}=\left(\begin{array}{cccc}
c_{0} & c_{1} & \ldots & c_{n} \\
c_{1} & c_{2} & \ldots & c_{n+1} \\
\vdots & \vdots & & \vdots \\
c_{n} & c_{n+1} & \ldots & c_{2 n}
\end{array}\right)
$$

is strongly regular, that is, $\operatorname{det}\left(H_{n}\right) \neq 0$ for all $n \geq 0$. Conversely, the ShohatFavard Theorem tells us that, for any linear functional $c$ with strongly regular sequence of Hankel matrices and normalization $c(1)=c_{0}=1$, there exists a sequence of formal orthogonal polynomials $\left(q_{n}(z)\right)_{n \geq 0}$ verifying the three term recurrence relation (2) and (3) for some complex Jacobi matrix.

Notice that a Jacobi matrix is real (i.e., $a_{n}, b_{n} \in \mathrm{R}$ ) if and only if all Hankel matrices are symmetric positive definite. Many equivalent conditions are known to characterize the uniqueness of the corresponding moment problem. For convenience we state some of them in the following theorem. Here P denotes the set of polynomials with complex coefficients.

THEOREM 1.1. For $a_{n}, b_{n} \in \mathrm{R}$, the following conditions are equivalent:

(A) There is a unique solution for the moment problem, that is, there is a unique positive probability measure $\mu$ supported on the real line such that the moments $c_{n}$ of (4) can be written as $c_{n}=\int x^{n} d \mu(x)$ for all $n \geq 0$.

(B) The Jacobi matrix $\mathscr{A}$ of (1) is determinate, that is, for any $z \in \mathrm{C}$, at least one of the sequences $p(z)$ and $q(z)$ is not in $\ell^{2}$.

(C) There is a unique closed extension in $\ell^{2}$ of the Jacobi matrix $\mathscr{A}$ which is defined by matrix product. 
(D) The linear operator $T: \mathrm{P} \mapsto \mathrm{P}$, defined by $T\left(x^{n}\right)=q_{n}, n \geq 0$, is an unbounded operator with respect to the norm $\|p\|^{2}=\frac{1}{2 \pi} \int_{|z|=1}|p(z)|^{2}|d z|$ of the Hardy space $H^{2}$.

(E) The inverses of the Hankel matrices $H_{n}, n \geq 0$, of (5) are not uniformly bounded.

(F) The numerical range of the Hankel matrices is not bounded away from zero: $\inf \left\{\left|\left(y, H_{n} y\right) /(y, y)\right|: y \in \mathrm{C}^{n}, n \geq 0\right\}=0$.

The equivalence of the first three properties is very classical, see for instance [1], [12] or the more recent paper [11, Theorem 2 and Theorem 3]. The equivalence of conditions (A) and (E) was the content of the recent BergChen-Ismael Theorem [5]. Since $H_{n}$ is symmetric and positive definite in the real case, both properties $(\mathrm{E})$ and $(\mathrm{F})$ are equivalent to the fact that the smallest eigenvalue of $H_{n}$ tends to zero as $n \rightarrow \infty$. For property (D) in more general settings we refer the reader to [6].

The aim of the present paper is to explore possible generalizations of Theorem 1.1 to the setting of complex Jacobi matrices. Condition (A) is out of the scope of the present paper. The notion of indeterminacy for complex Jacobi matrices (see property (B)) was introduced by Wall [13, Definition 22.1] who showed in the theorem of invariability [13, Theorem 22.1] that both $p(z)$ and $q(z)$ are elements of $\ell^{2}$ for all $z \in \mathrm{C}$ if this condition holds for just one $z \in \mathrm{C}$. With respect to condition $(\mathrm{C})$ we recall from $[3, \S 2.1]$ that there is a minimal and a maximal closed extension of $\mathscr{A}$ defined by matrix product, namely the operators $A=A_{\min }$ and $A_{\max }$, with $A_{\min }$ being the closure of $\mathscr{A}$, and thus

$$
\mathscr{D}\left(A_{\text {min }}\right)=\left\{y \in \ell^{2}: \exists\left(y^{(n)}\right)_{n \geq 0} \subset \mathscr{C}_{0}: y^{(n)} \underset{n}{\rightarrow} y,\left(\mathscr{A} y^{(n)}\right)_{n \geq 0} \underset{n}{\rightarrow} \mathscr{A} y\right\},
$$

whereas $D\left(A_{\max }\right)=\left\{y \in \ell^{2}: \mathscr{A} y \in \ell^{2}\right\}$. A complex Jacobi matrix is called proper [3, Definition 2.2] if these operators coincide, or, in other words, if condition (C) of Theorem 1.1 holds. Concerning the equivalence of conditions (B) and (C), it was shown in [3, Theorem 2.6(b)] that any proper complex Jacobi matrix is determinate. The converse was only shown to be true for Jacobi matrices with non-empty essential spectrum $\sigma_{\text {ess }}(A)$ [3, Theorem 2.6(b)], and remained an open question for general complex Jacobi matrices. In this context, recall from [3, Theorem 2.6(c)] that a Jacobi operator may only have spectrum $\sigma(A) \neq \mathrm{C}$ if $\mathscr{A}$ is proper. Also, for proper as well as indeterminate complex Jacobi matrices, the problem of characterizing the resolvent [3, Theorems 2.10 and 2.11] and the problem of finite section resolvent convergence [3, Theorems 4.1 and 4.2] are well understood.

The paper is organized as follows: In $\$ 2$ we establish the equivalence between proper and determinate Jacobi matrices, and draw some conclusions. 
In $\S 3$ we show that condition (D) implies property (B), and give classes of complex Jacobi matrices where the reciprocal is true. The other implications are discussed in $\S 4$.

\section{Proper and determinate Jacobi matrices}

The aim of this section is to prove the following statement.

THEOREM 2.1. A complex Jacobi matrix is proper iff it is determinate.

For an indeterminate Jacobi matrix $\mathscr{A}$ one may show that $\mathscr{N}\left(A_{\text {max }}\right) \neq\{0\}$ and that $\mathscr{R}\left(A_{\max }\right)$ is dense in $\ell^{2}$, implying that $\mathscr{A}$ is not proper (compare with [3, Theorem 2.6(b)]). Hence, according to [13, Theorem 22.1], we only have to show that for a complex Jacobi matrix with $A_{\min } \neq A_{\max }$ one has $p(0) \in \ell^{2}$ and $q(0) \in \ell^{2}$.

Before entering into details, let us mention the following consequence answering a question raised in [2, Remark 1].

Corollary 2.2. Let $\mathscr{A}$ and $\tilde{\mathscr{A}}$ be two complex Jacobi matrices, with $\mathscr{A}-\widetilde{\mathscr{A}}$ being bounded (that is, $\sup _{n}\left|a_{n}-\widetilde{a}_{n}\right|+\sup _{n}\left|b_{n}-\widetilde{b}_{n}\right|<\infty$ ). Then $\mathscr{A}$ is indeterminate iff $\tilde{\mathscr{A}}$ is indeterminate.

The preservation of indeterminacy under diagonal bounded perturbations has been discussed in [13, Theorem 22.1]. For a real Jacobi matrix $\mathscr{A}$, the statement of Corollary 2.2 has already been obtained in [7] (though not stated there, the techniques presented in [7] do cover a more general frame). A proof of Corollary 2.2 is based on Theorem 2.1 and the observation that a proper Jacobi matrix remains proper after bounded perturbations (since the domains of definition of $A_{\max }$ and $A_{\min }$ remain invariant).

Example 2.3. Let $\left(b_{n}\right)_{n \geq 0}$ be bounded. Then $\mathscr{A}$ is indeterminate iff

$$
\sum_{n=0}^{\infty}\left|\prod_{j=0}^{n} \frac{a_{2 j}}{a_{2 j+1}}\right|^{2}+\sum_{n=0}^{\infty}\left|\prod_{j=0}^{n} \frac{a_{2 j+1}}{a_{2 j+2}}\right|^{2}<\infty .
$$

Moreover, the finiteness of the sums is invariant under bounded perturbation of the $a_{n}$. In order to prove these two statements, it is sufficient to consider only the case $b_{n}=0$ for all $n$ by Corollary 2.2. In this case, we find that $p_{2 n}(0)=q_{2 n+1}(0)=0$ for all $n \geq 0$, whereas for $q_{2 n}(0)$ and $p_{2 n+1}(0)$ we essentially obtain the products mentioned in the above formula.

Returning to the proof of Theorem 2.1, let $\mathscr{A}$ be a complex Jacobi matrix 
with $A_{\min } \neq A_{\max }$, and consider the block tridiagonal matrix

$$
\mathscr{B}=\left(\begin{array}{cccc}
B_{0} & A_{0} & 0 & \ldots \\
A_{0} & B_{1} & A_{1} & \ddots \\
0 & A_{1} & B_{2} & \ddots \\
\vdots & \ddots & \ddots & \ddots
\end{array}\right),
$$

where

$$
A_{k}=\left(\begin{array}{cc}
0 & a_{k} \\
\overline{a_{k}} & 0
\end{array}\right)=A_{k}^{*}, \quad B_{k}=\left(\begin{array}{cc}
0 & b_{k} \\
b_{k} & 0
\end{array}\right)=B_{k}^{*} .
$$

Notice that $\mathscr{B}$ acting on $\mathscr{C}_{0}$ via matrix product is now a symmetric operator (since its matrix representation is hermitian). As before, we may consider $B:=$ $B_{\text {min }}$, the closure of $\mathscr{B}$, as well as $B_{\max }$ with $\mathscr{D}\left(B_{\max }\right)=\left\{y \in \ell^{2}: \mathscr{B} \cdot y \in \ell^{2}\right\}$, the maximal closed operator defined by matrix product, see also $[3, \S 2.1]$. It follows from [3, Lemma 2.1] that $B_{\max }=B^{*}$.

The statement of Theorem 1.1 can be generalized to the setting of hermitian block Jacobi matrices. For the sake of completeness, we will show in Lemma 2.4 and Lemma 2.5 below those implications which are required for our proof of Theorem 2.1.

Lemma 2.4. Let $\mathscr{A}$ be a complex Jacobi matrix with $A_{\min } \neq A_{\max }$, and consider the $2 \times 2$ matrix polynomials being defined by $(n=0,1,2, \ldots)$

$$
\begin{gathered}
A_{n} Q_{n+1}(z)+B_{n} Q_{n}(z)+A_{n-1} Q_{n-1}(z)=z Q_{n}(z), \\
A_{n} P_{n+1}(z)+B_{n} P_{n}(z)+A_{n-1} P_{n-1}(z)=z P_{n}(z), \\
Q_{-1}(z)=0, \quad Q_{0}(z)=I_{2}, \quad P_{0}(z)=0, \quad P_{1}(z)=A_{0}^{-1} .
\end{gathered}
$$

Then, for each purely imaginary $z \in i \cdot \mathbf{R} \backslash\{0\}$,

$$
\sum_{n=0}^{\infty}\left\|P_{n}(z)\right\|^{2}+\sum_{n=0}^{\infty}\left\|Q_{n}(z)\right\|^{2}<\infty .
$$

Proof. First, after a suitable simultaneous permutation of rows and columns (we first take the even indices), the matrix $\mathscr{B}$ becomes the matrix

$$
\left(\begin{array}{cc}
0 & \mathscr{A} \\
\mathscr{A} & 0
\end{array}\right)
$$

$\overline{\mathscr{A}}$ obtained from $\mathscr{A}$ by conjugating each entry. It follows that

$$
\begin{aligned}
\mathscr{D}(B)= & \left\{\left(y_{n}\right)_{n \geq 0} \in \ell_{2}:\left(y_{2 k+1}\right)_{k \geq 0},\left(\overline{y_{2 k}}\right)_{k \geq 0} \in \mathscr{D}\left(A_{\min }\right)\right\} \\
& \neq \mathscr{D}\left(B^{*}\right)=\left\{\left(y_{n}\right)_{n \geq 0} \in \ell_{2}:\left(y_{2 k+1}\right)_{k \geq 0},\left(\overline{y_{2 k}}\right)_{k \geq 0} \in \mathscr{D}\left(A_{\max }\right)\right\} .
\end{aligned}
$$


Hence $B$ is not self-adjoint. Fix $z \in i \cdot \mathbf{R} \backslash\{0\}$ as in the assertion of the Lemma. We consider the reduced modulus $[8, \S$ IV.5.1]

$$
\gamma(B)=\min _{y \in \mathscr{D}(B)} \frac{\|(z I-B) y\|}{\operatorname{dist}(y, \mathcal{N}(z I-B))} .
$$

One easily obtains the lower bound

$$
\begin{aligned}
\gamma(B) & \geq \min _{y \in \mathscr{D}(B)} \frac{\|(z I-B) y\|}{\|y\|}=\min _{y \in \mathscr{C}_{0}} \frac{\|(z I-B) y\|}{\|y\|} \\
& =\min _{y \in \mathscr{C}_{0}} \sqrt{|\operatorname{Im}(z)|^{2}+\frac{(B y, B y)}{(y, y)}} \geq|z|>0 .
\end{aligned}
$$

As a consequence, $\mathscr{N}(z I-B)=\{0\}$, and both images $\mathscr{R}(z I-B)$ and $\mathscr{R}\left((z I-B)^{*}\right)=\mathscr{R}\left(\bar{z} I-B^{*}\right)$ are closed according to [8, Theorem IV.5.13]. In particular, with $\bar{z}=-z$ replacing $z$ we obtain

$$
\mathscr{R}\left(z I-B^{*}\right)=\mathscr{N}(\bar{z} I-B)^{\perp}=\ell^{2} .
$$

We claim that $\mathcal{N}\left(z I-B^{*}\right) \neq\{0\}$. Indeed, otherwise $\mathscr{R}(z I-B)=\mathscr{N}(\bar{z} I-$ $\left.B^{*}\right)^{\perp}=\ell^{2}$, and both $z I-B$ and $z I-B^{*}$ are injective, a contradiction to the fact that $B$ is not self-adjoint, i.e., that $B^{*}$ is a proper extension of $B$.

We may even determine the form of $\mathcal{N}\left(z I-B^{*}\right)$. By writing down explicitly the matrix product and comparing it with the above recurrence relations for $\left(Q_{n}(z)\right)_{n \geq 0}$, we see that for any solution of $\left(z I-B^{*}\right) y=0$ there necessarily exist a vector $d \in \mathrm{C}^{2}$ with $y=\left(Q_{n}(z) d\right)_{n \geq 0} \in \ell^{2}$. Hence $1 \leq \operatorname{dim} \mathcal{N}(z I-$ $\left.B^{*}\right) \leq 2$. However, $y \in \mathcal{N}\left(z I-B^{*}\right)$ if and only if

$$
z y^{\prime}=\mathscr{A} y^{\prime \prime}, \quad z y^{\prime \prime}=\overline{\mathscr{A}} y^{\prime},
$$

where $y^{\prime}=\left(y_{2 k}\right)_{k \geq 0} \in \ell^{2}$ and $y^{\prime \prime}=\left(y_{2 k+1}\right)_{k \geq 0} \in \ell^{2}$. Taking conjugates leads to $-z \overline{y^{\prime}}=\bar{z} \overline{y^{\prime}}=\overline{\mathscr{A}} \overline{y^{\prime \prime}}$ and $-z \overline{y^{\prime \prime}}=\bar{z} \overline{y^{\prime \prime}}=\mathscr{A} \overline{y^{\prime \prime}}$. Consequently, with $0 \neq y \in \mathcal{N}\left(z I-B^{*}\right)$, the orthogonal vector $\tilde{y}$ defined by $\tilde{y}_{2 k+1}=-\overline{y_{2 k}}, \tilde{y}_{2 k}=$ $\overline{y_{2 k+1}}$ is also an element of $\mathcal{N}\left(z I-B^{*}\right)$, showing that $\operatorname{dim} \mathcal{N}\left(z I-B^{*}\right)=2$, i.e., the block Jacobi matrix $\mathscr{B}$ has the deficiency indices $(2,2)$. Thus both columns of $Q(z):=\left(Q_{n}(z)\right)_{n \geq 0}$ are elements of $\ell^{2}$. In addition, denoting by $\|\cdot\|_{F}$ the Froebenius norm, we have that

$$
\begin{aligned}
\sum_{n=0}^{\infty}\left\|Q_{n}(z)\right\|^{2} \leq \sum_{n=0}^{\infty}\left\|Q_{n}(z)\right\|_{F}^{2} & =\sum_{n=0}^{\infty}\left\|Q_{n}(z) \cdot\left(\begin{array}{l}
1 \\
0
\end{array}\right)\right\|^{2}+\left\|Q_{n}(z) \cdot\left(\begin{array}{l}
0 \\
1
\end{array}\right)\right\|^{2} \\
& =\left\|Q(z)\left(\begin{array}{l}
1 \\
0
\end{array}\right)\right\|^{2}+\left\|Q(z)\left(\begin{array}{l}
0 \\
1
\end{array}\right)\right\|^{2}<\infty,
\end{aligned}
$$


which is the first part of the assertion of the Lemma. In order to show the second part, recall from above that $e_{0}, e_{1} \in \mathscr{R}\left(z I-B^{*}\right)$. Again by writing down explicitly the matrix product for the pre-images of $\left(e_{0}, e_{1}\right)$ and comparing it with the above recurrence relations for $\left(P_{n}(z)\right)_{n \geq 0}$ and $\left(Q_{n}(z)\right)_{n \geq 0}$, we see that there exists a $D \in \mathrm{C}^{2 \times 2}$ with $\left(z I-B^{*}\right) Y=\left(e_{0}, e_{1}\right)$ and $Y=$ $\left(Q_{n}(z) D-P_{n}(z)\right)_{n \geq 0} \in\left(\ell^{2}\right)^{2}$. Consequently, both columns of $\left(P_{n}(z)\right)_{n \geq 0}$ are also elements of $\ell^{2}$, and we obtain as above the relation $\sum_{n=0}^{\infty}\left\|P_{n}(z)\right\|^{2}<\infty$.

It is not difficult to see that (7) implies that

$$
\lim _{n \rightarrow \infty}\left(\sum_{n=0}^{\infty} Q_{n}(z)^{*} Q_{n}(z)\right)^{-1}
$$

exists and is invertible for any $z \in i \cdot \mathbf{R} \backslash\{0\}$. It therefore follows from [9, Theorem 3.1] (see also [4, Theorem 2.6, p. 570]) that this limit exists and is invertible for any $z \in C \backslash R$. Such a case is usually referred to as the completely indeterminate case.

By comparing the two recurrence relations, one easily verifies that

$$
Q_{n}(0)=\left(\begin{array}{cc}
\overline{q_{n}(0)} & 0 \\
0 & q_{n}(0)
\end{array}\right), \quad P_{n}(0)=\left(\begin{array}{cc}
0 & \overline{p_{n}(0)} \\
p_{n}(0) & 0
\end{array}\right), \quad n \geq 0 .
$$

Hence, if we are able to show that (7) also holds for $z=0$, then the indeterminate case holds for $\mathscr{A}$, as claimed in Theorem 2.1.

Similar to the scalar case one may give a theorem of invariability in the matrix setting, compare for instance with the related result [9, Theorem 3.2]. For our purpose it is sufficient to show the following result.

Lemma 2.5. Let $\mathscr{A}$ be a complex Jacobi matrix with $A_{\min } \neq A_{\max }$. Then, for each $z \in \mathrm{C}$,

$$
\sum_{n=0}^{\infty}\left\|P_{n}(z)\right\|^{2}+\sum_{n=0}^{\infty}\left\|Q_{n}(z)\right\|^{2}<\infty
$$

Proof. Denote by $Q_{n}^{*}(z)$ (respectively $P_{n}^{*}(z)$ ), the $2 \times 2$ matrix polynomial $Q_{n}(\bar{z})^{*}$ (respectively $\left.P_{n}(\bar{z})^{*}\right)$, and by $\mathscr{B}_{2 n+2}$ the principal submatrix of order $2 n+2$ of the matrix $\mathscr{B}$ of (6). One easily shows by recurence on $n$

$$
\left(\begin{array}{ll}
P_{n}^{*}(z) & P_{n+1}^{*}(z) \\
Q_{n}^{*}(z) & Q_{n+1}^{*}(z)
\end{array}\right) \cdot\left(\begin{array}{cc}
0 & -A_{n} \\
A_{n} & 0
\end{array}\right) \cdot\left(\begin{array}{cc}
Q_{n}(z) & -P_{n}(z) \\
Q_{n+1}(z) & -P_{n+1}(z)
\end{array}\right)=I_{4},
$$


and the recurrence relations

$$
\begin{aligned}
\left(z I_{2 n+2}-\mathscr{B}_{2 n+2}\right)\left(\begin{array}{c}
Q_{0}(z) \\
\vdots \\
Q_{n}(z)
\end{array}\right) & =\left(\begin{array}{c}
0 \\
\vdots \\
0 \\
A_{n} Q_{n+1}(z)
\end{array}\right), \\
\left(Q_{0}^{*}(x), \ldots, Q_{n}^{*}(x)\right)\left(x I_{2 n+2}-\mathscr{B}_{2 n+2}\right) & =\left(0, \ldots, 0, Q_{n+1}^{*}(x) A_{n}\right), \\
\left(P_{0}^{*}(x), \ldots, P_{n}^{*}(x)\right)\left(x I_{2 n+2}-\mathscr{B}_{2 n+2}\right)= & \left(-I_{2}, 0, \ldots, 0, P_{n+1}^{*}(x) A_{n}\right) .
\end{aligned}
$$

The last two relations together with (10) give

$$
\begin{array}{r}
\left(Q_{n}(x)\left(P_{0}^{*}(x), \ldots, P_{n}^{*}(x)\right)-P_{n}(x)\left(Q_{0}^{*}(x), \ldots, Q_{n}^{*}(x)\right)\right)\left(x I_{2 n+2}-\mathscr{B}_{2 n+2}\right) \\
=\left(-Q_{n}(x), 0, \ldots, 0, I_{2}\right),
\end{array}
$$

where we notice that, by (10), the last block component of the vector on the left-hand side vanishes. Multiplying this relation on the right by the first components of $Q(z)$ gives for $x \neq z$,

$$
Q_{n}(z)-Q_{n}(x)=(x-z) \sum_{k=0}^{n-1}\left[Q_{n}(x) P_{k}^{*}(x) Q_{k}(z)-P_{n}(x) Q_{k}^{*}(x) Q_{k}(z)\right] .
$$

Let us write this relation as $Q(z)=Q(x)+(x-z) \mathscr{H} Q(z)$, a formal product between infinite matrices, with $\mathscr{H}$ having non-zero entries only below the main diagonal. Choosing $x \in i \cdot \mathbf{R} \backslash\{0\}$ and $z \in \mathbf{C}$, we observe that

$$
\begin{aligned}
\sum_{n, k}\left|\mathscr{H}_{n, k}\right|^{2} & =\sum_{n>k}\left\|Q_{n}(x) P_{k}^{*}(x)-P_{n}(x) Q_{k}^{*}(x)\right\|_{F}^{2} \\
& \leq 4 \sum_{n, k}\left\|Q_{n}(x)\right\|^{2} \cdot\left\|P_{k}^{*}(x)\right\|^{2}+\left\|P_{n}(x)\right\|^{2} \cdot\left\|Q_{k}^{*}(x)\right\|^{2} \\
& =4 \sum_{n, k}\left\|Q_{n}(x)\right\|^{2} \cdot\left\|P_{k}(-x)\right\|^{2}+\left\|P_{n}(x)\right\|^{2} \cdot\left\|Q_{k}(-x)\right\|^{2},
\end{aligned}
$$

the last expression being finite by Lemma 2.4. Since $Q(x)$ has columns in $\ell^{2}$, we may conclude from [10, Lemma II.7.3] that that the columns of $Q(z)$ are in $\ell^{2}$. Finally, for $P(z)$ one first establishes the formula

$$
P_{n}(z)-P_{n}(x)=(x-z) \sum_{k=0}^{n-1}\left[Q_{n}(x) P_{k}^{*}(x) P_{k}(z)-P_{n}(x) Q_{k}^{*}(x) P_{k}(z)\right]
$$

and then concludes in the same manner. 


\section{Indeterminacy and the map $T$}

In this section we relate the determinacy of a complex Jacobi matrix to property (D) of Theorem 1.1. Recall that the linear map $T$ was defined on the space $\mathrm{P}$ of polynomials via the relation $T\left(z^{n}\right)=q_{n}$. We write $q_{n}(z)=$ $q_{0, n}+q_{1, n} z+\ldots+q_{n, n} z^{n}$, and consider the triangular matrix

$$
T_{n}=\left(\begin{array}{ccccc}
q_{00} & q_{01} & q_{02} & \ldots & q_{0 n} \\
0 & q_{11} & q_{12} & & \vdots \\
0 & 0 & q_{22} & & \\
\vdots & & \ddots & \ddots & \vdots \\
0 & \ldots & 0 & & q_{n n}
\end{array}\right) .
$$

Notice that $T_{n}$ is a matrix description of the restriction of the application $T$ to the set $\mathbf{P}_{n}$ of polynomials of degree at most $n$ : for $p(z)=a_{0}+a_{1} z+\cdots+a_{n} z^{n}$ we have $(T p)(z)=a_{0} q_{0}(z)+a_{1} q_{1}(z)+\cdots+a_{n} q_{n}(z)=b_{0}+b_{1} z+\cdots+b_{n} z^{n}$, with

$$
b=T_{n} a, \quad a=\left(a_{0}, \ldots, a_{n}\right)^{t}, \quad b=\left(b_{0}, \ldots, b_{n}\right)^{t} .
$$

Since in addition the Hardy space norm $\|p\|_{H^{2}}$ of a polynomial $p(z)=a_{0}+$ $a_{1} z+\cdots+a_{n} z^{n}$ coincides with the euclidean norm $\|a\|$ of the corresponding vector of coefficients $a=\left(a_{0}, \ldots, a_{n}\right)^{t}$, we may conclude that

$$
\left\|\left.T\right|_{\mathbf{P}_{n}}\right\|_{H^{2} \mapsto H^{2}}=\sup _{p \in \mathrm{P}_{n}} \frac{\|T p\|_{H^{2}}}{\|p\|_{H^{2}}}=\sup _{a \in \mathrm{C}^{n+1}} \frac{\left\|T_{n} a\right\|}{\|a\|}=\left\|T_{n}\right\| .
$$

As a consequence, we get the following expression for the Hardy space operator norm of $T$

$$
\|T\|_{H^{2} \mapsto H^{2}}=\sup _{n \geq 0}\left\|T_{n}\right\| .
$$

For real Jacobi matrices we know from Theorem 1.1 that $\mathscr{A}$ is indeterminate if and only if the quantity $\|T\|_{H^{2} \mapsto H^{2}}$ is finite. As we show in Theorem 3.1 below, one implication of this assertion remains valid for complex Jacobi matrices $\mathscr{A}$. However, we are only able to prove the other implication under additional assumptions on $\mathscr{A}$ (compare also with Corollary 3.2 and Theorem 3.3 below).

THEOREM 3.1. If $\mathscr{A}$ is an indeterminate complex Jacobi matrix then $\|T\|_{H^{2} \mapsto H^{2}}<\infty$. 
Conversely, a complex Jacobi matrix is indeterminate provided that $\|T\|_{H^{2} \mapsto H^{2}}<\infty$, and in addition ( $\mathrm{D}$ denoting the open unit disk)

$$
\exists \zeta \in \mathrm{D}: \sup _{y \in \mathscr{C}_{0}} \frac{\|y\|}{\|(\zeta I-A) y\|}<\infty .
$$

Proof. We start by establishing the following formulas (compare with [5], [6])

$$
\left\|T_{n}\right\|^{2} \leq\left\|T_{n}\right\|_{F}^{2}=\frac{1}{2 \pi} \int_{0}^{2 \pi} \sum_{j=0}^{n}\left|q_{j}\left(e^{i t}\right)\right|^{2} d t,
$$

$\|\cdot\|_{F}$ denoting the Froebenius (or Hilbert-Schmidt) norm, and, for $z \in \mathrm{D}$,

$$
\sum_{j=0}^{n}\left|q_{j}(z)\right|^{2} \leq \frac{1}{1-|z|^{2}} \cdot\left\|T_{n}\right\|^{2} .
$$

Formula (14) follows from the following simple observation

$$
\begin{aligned}
\left\|T_{n}\right\|^{2} \leq\left\|T_{n}\right\|_{F}^{2} & =\sum_{k=0}^{n} \sum_{j=0}^{n}\left|\left(T_{n}\right)_{j, k}\right|^{2}=\sum_{k=0}^{n} \sum_{j=0}^{k}\left|q_{j, k}\right|^{2} \\
& =\sum_{k=0}^{n} \frac{1}{2 \pi} \int_{0}^{2 \pi}\left|q_{k}\left(e^{i t}\right)\right|^{2} d t .
\end{aligned}
$$

For a proof of (15), notice that, for $z \in \mathrm{D}$,

$$
\begin{aligned}
\sum_{j=0}^{n}\left|q_{j}(z)\right|^{2} & =\left\|\left(q_{0}(z), \ldots, q_{n}(z)\right)\right\|^{2}=\left\|\left(1, z, \ldots, z^{n}\right) T_{n}\right\|^{2} \\
& \leq\left\|T_{n}\right\|^{2} \cdot\left\|\left(1, z, \ldots, z^{n}\right)\right\|^{2} \leq \frac{1}{1-|z|^{2}} \cdot\left\|T_{n}\right\|^{2} .
\end{aligned}
$$

Suppose now that $\mathscr{A}$ is indeterminate. We remark that, according to the theorem of invariability [13, Theorem 22.1], the series $\sum_{j=0}^{\infty}\left|q_{j}(z)\right|^{2}$ converges uniformly on compact sets in the indeterminate case. As a consequence, the function $z \mapsto\|q(z)\|_{\ell^{2}}$ mapping $C$ to $(0,+\infty)$ is continuous, and $\|T\|_{H^{2} \mapsto H^{2}}<\infty$ is a consequence of (12) and (14).

Conversely, suppose that $\|T\|_{H^{2} \mapsto H^{2}}<\infty$, and let $\zeta \in \mathrm{D}$ be as in the statement of the Theorem. Then from (12) and (15) we get $q(\zeta) \in \ell^{2}$, and thus $q(\zeta) \in \mathscr{N}\left(\zeta I-A_{\max }\right)$. On the other hand, $q(\zeta) \in \mathcal{N}\left(\zeta I-A_{\min }\right)$ implies that there is a sequence $\left(v_{n}\right) \subset \mathscr{C}_{0}$, with $v_{n} \rightarrow q(\zeta)$ and $(\zeta I-A) v_{n} \rightarrow$ 
0 for $n \rightarrow \infty$, in contradiction with the assumption on $\zeta$. Consequently, $\mathscr{N}\left(\zeta I-A_{\max }\right) \neq \mathcal{N}\left(\zeta I-A_{\min }\right)$. Thus, $\mathscr{A}$ is not proper, and the indeterminacy follows from Theorem 2.1.

In the indeterminate case, all principal submatrices $(\zeta I-\mathscr{A})_{n+1, n}$ of $\zeta I-$ $\mathscr{A}$ of size $(n+2) \times(n+1)$ can be shown to have a bounded left inverse, implying that condition (13) holds for all $\zeta \in \mathrm{C}$. A simple sufficient condition for (13) is that there exists an infinite set $\Lambda$ such that the sequence ([( $\zeta I-$ $\left.\left.\mathscr{A})_{n, n}\right]^{-1}\right)_{n \in \Lambda}$, of inverses of finite subsections of $\zeta I-\mathscr{A}$, is bounded. A class of complex Jacobi matrices having property (13) was discussed by Wall: the complex Jacobi matrix $\mathscr{A}$ is called positive definite if its numerical range satisfies $\Theta\left(A_{\min }\right) \subset\{\operatorname{Im}(w) \leq 0\}$. We remind the reader that, for a linear operator $S$ defined on some Hilbert space, the numerical range (or field of values) is defined by

$$
\Theta(S):=\{(y, S y): y \in \mathscr{D}(S),\|y\|=1\},
$$

see, e.g., [8, Section V. 3.2].

Corollary 3.2. A positive definite complex Jacobi matrix is indeterminate if and only if $\|T\|_{H^{2} \mapsto H^{2}}<\infty$.

Proof. We only need to show that (13) holds for all $\zeta \in \mathrm{C}$ with $\operatorname{Im}(\zeta)>0$. Indeed, we have for all $y \in \mathscr{C}_{0}$

$$
\frac{\|y\|}{\|(\zeta I-A) y\|} \leq \frac{\|y\|^{2}}{|(y,(\zeta I-A) y)|} \leq \frac{1}{\operatorname{dist}\left(\zeta, \Theta\left(A_{\min }\right)\right)} \leq \frac{1}{\operatorname{Im}(\zeta)}<\infty .
$$

In order to complete the discussion about the equivalence of properties (B) and (D) of Theorem 1.1 for complex Jacobi matrices, we show in Theorem 3.3 below that the additional assumption (13) of Theorem 3.1 can be replaced by (17). We make some preliminary remarks in order to motivate this new condition. The orthogonality relation $c\left(q_{j} q_{k}\right)=\delta_{j, k}$ may be rewritten as $T_{n}^{t} H_{n} T_{n}=I_{n+1}\left({ }^{t}\right.$ denoting the transposed without taking conjugates), or

$$
H_{n}^{-1}=T_{n} T_{n}^{t}
$$

Notice also that $\left(1, x, \ldots, x^{n}\right) T_{n}=\left(q_{0}(x), \ldots, q_{n}(x)\right)$, and thus

$$
\left(1, x, \ldots, x^{n}\right) H_{n}^{-1}\left(1, y, \ldots, y^{n}\right)^{t}=\sum_{j=0}^{n} q_{j}(x) q_{j}(y)=: K_{n}(x, y) .
$$

If now $\|T\|_{H^{2} \mapsto H^{2}}<\infty$, then $q(x), q(y) \in \ell^{2}$ by (15), and hence $K_{n}(x, y)$ tends to some function $K(x, y)$ for $n \rightarrow \infty$ uniformly on compact subsets of 
$\mathrm{D} \times \mathrm{D}$. In particular, $K$ is analytic in both arguments. As a consequence, $\lim _{n \rightarrow \infty}\left(e_{j}, H_{n}^{-1} e_{k}\right)=\left(e_{j}, T T^{t} e_{k}\right)=\left.\frac{1}{j !}\left(\frac{d}{d x}\right)^{j} \frac{1}{k !}\left(\frac{d}{d y}\right)^{k} K(x, y)\right|_{(x, y)=(0,0)}$.

Thus $K(x, y)=0$ for all $x, y \in \mathrm{D}$ if and only if the $(j, k)$ entry of $H_{n}^{-1}$ tends to zero for all $j, k$, if and only if the bounded operator $T T^{t}$ is the zero operator. Clearly, this last property cannot be true for the case of real Jacobi matrices (since here $\left.K(0,0) \geq q_{0}(0)^{2}=1\right)$, but there remains the open question whether $T T^{t}=0$ can be true for complex Jacobi matrices. ${ }^{1}$

Theorem 3.3. Let $\mathscr{A}$ be a complex Jacobi matrix for which there exist $x, y \in \mathrm{D}$ with

$$
\limsup _{n \rightarrow \infty}\left|\sum_{j=0}^{n} q_{j}(x) q_{j}(y)\right|>0 .
$$

Then $\mathscr{A}$ is indeterminate if and only if $\|T\|_{H^{2} \mapsto H^{2}}<\infty$.

Proof. We only need to consider the case $\|T\|_{H^{2} \mapsto H^{2}}<\infty$, and hence only the case $K(x, y) \neq 0$. Notice that

$$
\begin{aligned}
\left(1, z, \ldots, z^{n+1}\right) T_{n+1}(x I-\mathscr{A})_{n+1, n} & =\left(q_{0}(z), \ldots, q_{n+1}(z)\right)(x I-\mathscr{A})_{n+1, n} \\
& =(x-z)\left(1, z, \ldots, z^{n}\right) T_{n} .
\end{aligned}
$$

Comparing powers of $z$ we obtain

$$
T_{n+1}(x I-\mathscr{A})_{n+1, n}=M_{n}(x) T_{n},
$$

where

$$
M_{n}(x):=\left[\begin{array}{cccc}
x & 0 & \cdots & 0 \\
-1 & \ddots & \ddots & \vdots \\
0 & \ddots & \ddots & 0 \\
\vdots & \ddots & \ddots & x \\
0 & \cdots & 0 & -1
\end{array}\right],
$$

with $\left\|M_{n}(x) v\right\| \geq(1-|x|)\|v\|$ for any vector $v$. Consequently,

$$
0<\frac{1-|x|}{\|T\|_{H^{2} \mapsto H^{2}}} \leq(1-|x|) \min _{n} \min _{v \in \mathrm{C}^{n+1}} \frac{\left\|T_{n+1}^{-1} v\right\|}{\|v\|}
$$

\footnotetext{
${ }^{1}$ If the answer to this question would be no, then the condition (17) of Theorem 3.3 could be dropped, i.e., there would be equivalence between the properties (B) and (D) of Theorem 1.1 for complex Jacobi matrices.
} 


$$
\begin{gathered}
\leq(1-|x|) \min _{n} \min _{v \in \mathrm{C}^{n}} \frac{\left\|T_{n+1}^{-1} M_{n}(x) v\right\|}{\left\|M_{n}(x) v\right\|} \leq \min _{n} \min _{v \in \mathrm{C}^{n}} \frac{\left\|T_{n+1}^{-1} M_{n}(x) v\right\|}{\|v\|} \\
=\min _{n} \min _{v \in \mathrm{C}^{n}} \frac{\left\|(x I-\mathscr{A})_{n+1, n} v\right\|}{\left\|T_{n} v\right\|}=\min _{v \in \mathscr{C}_{0}} \frac{\|(x I-A) v\|}{\|T v\|} \\
\leq \frac{1}{\sqrt{1-\left|y^{2}\right|}} \min _{v \in \mathscr{C}_{0}} \frac{\|(x I-A) v\|}{\left|\left(1, y, y^{2}, \ldots\right) T v\right|} .
\end{gathered}
$$

As before we get $q(x) \in \ell^{2}$ from (15), and thus $q(x) \in \mathcal{N}\left(x I-A_{\text {max }}\right)$. If $\mathscr{A}$ is determinate, then it is also proper by Theorem 2.1 , and hence $q(x) \in$ $\mathcal{N}\left(x I-A_{\min }\right)$. As a consequence, there is a sequence $\left(v_{n}\right) \subset \mathscr{C}_{0}$, with $\|(x I-$ $A) v_{n} \| \rightarrow 0$ and $v_{n} \rightarrow q(x)$ for $n \rightarrow \infty$. Consequently,

$$
\left|\left(1, y, y^{2}, \ldots\right) T v_{n}\right|=\left|\left(q_{0}(y), q_{1}(y), \ldots\right) v_{n}\right| \rightarrow|K(x, y)| \neq 0,
$$

a contradiction to the above chain of inequalities. Hence $\mathscr{A}$ is indeterminate, as claimed in the statement of Theorem 3.3.

\section{Theorem 1.1 revisited for complex Jacobi matrices}

In $\S 2$ we have seen that the properties $(\mathrm{B})$ and $(\mathrm{C})$ of Theorem 1.1 remain equivalent for complex Jacobi matrices. Also, property (D) implies property (B) by Theorem 3.1, and we have given large classes of complex Jacobi matrices in $\$ 3$ where also the reciprocal is true. The following example shows that property $(\mathrm{F})$ no longer implies property $(\mathrm{B})$.

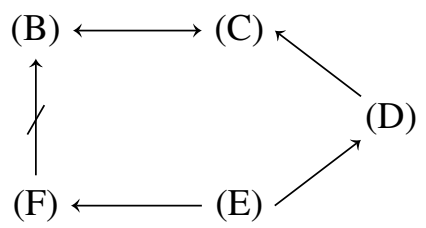

FIGURE 1. The implications of Theorem 1.1 remaining valid for complex Jacobi matrices.

Example 4.1. Let $\mathscr{A}$ be any indeterminate complex Jacobi matrix. From, e.g., Corollary 2.2 we see that $\mathscr{A}$ remains indeterminate after changing $a_{0}$ and $b_{0}$. For $a_{0}=i, b_{0}=-1$ we have

$$
c_{0}=1, \quad c_{1}=\left(e_{0}, \mathscr{A} e_{0}\right)=b_{0}=-1, \quad c_{2}=\left(e_{0}, \mathscr{A}^{2} e_{0}\right)=b_{0}^{2}+a_{0}^{2}=0,
$$

and

$$
H_{1}=\left(\begin{array}{rr}
1 & -1 \\
-1 & 0
\end{array}\right)
$$


has the eigenvalues $(1 \pm \sqrt{3}) / 2$, of different sign. Taking into account that $H_{1}$ is a principal minor, we get for all $n \geq 1$

$$
0 \in \operatorname{conv}\left(\sigma\left(H_{1}\right)\right)=\Theta\left(H_{1}\right) \subset \Theta\left(H_{n}\right),
$$

i.e., property $(\mathrm{F})$ of Theorem 1.1 is true.

From (16) we obtain $\left\|H_{n}^{-1}\right\| \leq\left\|T_{n}\right\|^{2}$. As a consequence, property (E) implies property (D), but we do not know whether the reciprocal is false. Finally, notice also that property (E) trivially implies property (F) (but not the reciprocal, see Example 4.1).

\section{REFERENCES}

1. Akhiezer, N. I., The Classical Moment Problem, Oliver \& Boyd, London, 1965.

2. Barrios, D., López, G., Martínez, A., and Torrano, E., On the domain of convergence and poles of complex J-fractions, J. Approx. Theory 93 (1998), 177-200.

3. Beckermann, B., Complex Jacobi matrices, J. Comput. Appl. Math. 127 (2001), 17-65.

4. Berezanskii, Ju. M., Expansions in eigenfunctions of selfadjoint operators, Transl. Math. Monogr., 1968.

5. Berg, C., Chen, Y., and Ismail, M., Small eigenvalues of large Hankel matrices: The indeterminate case, Math. Scand. 91 (2002), 67-81.

6. Berg, C., and Durán, A., Orthogonal polynomials and analytic functions associated to positive definite matrices, preprint.

7. Castro Smirnova, M., Determinacy of bounded complex perturbations of Jacobi matrices, J. Approx. Theory 104 (2000), 345-351.

8. Kato, T., Perturbation Theory for Linear Operators, Springer-Verlag, Berlin, 1966.

9. López-Rodríguez, P., The Nevanlinna Parametrization for a matrix moment problem, Math. Scand. 89 (2001), 245-267.

10. Nikishin, E. M., and Sorokin, V. N., Rational Approximations and Orthogonality, Transl. Math. Monogr. 92, 1991.

11. Simon, B., The classical moment problem as a self-adjoint finite difference operator, Adv. Math. 137 (1998), 82-203.

12. Shohat, J. A., and Tamarkin, J. D., The problem of Moments, American Mathematical Society, 1943.

13. Wall, H. S., Analytic Theory of Continued Fractions, Chelsea, New York, 1973.

LABORATOIRE DE MATHÉMATIQUES APPLIQUÉES FRE 2222 (ANO)

UFR MATHÉMATIQUES - M3

UST LILLE, F-59655 VILLENEUVE D'ASCQ CEDEX

FRANCE

E-mail: bbecker@ano.univ-lille1.fr

UNIVERSITY OF SEVILLA

APDO 1160

41080, SEVILLA

SPAIN

E-mail:mirta@us.es 